\title{
A study on student perception on a formative 'mini-viva' examination in Anatomy
}

Ilangarathne IPSD ${ }^{1}$, Dissanayake $\mathrm{CS}^{1}$, Rodrigo $\mathrm{PWDCH}^{1}$, De Silva $\mathrm{CL}^{1}$, Koralegedara $\mathrm{KIS}^{1}$, Jayawardana SMA ${ }^{1}$, Paththinige $\mathrm{CS}^{1}$

${ }^{I}$ Department of Anatomy, Faculty of Medicine and Allied Sciences, Rajarata University of Sri Lanka.

*dineshilangarathna23@gmail.com

Using assessments 'for learning' is achieved by providing opportunities to students to reflect on self, receive feedback and advice on improvement. This study evaluated students' perception on a formative 'mini-viva' examination in Anatomy conducted with this purpose. Mid-semester vivavoce examination was held for first year students of Faculty of Medicine, Rajarata University, at the end of the 'Regional Anatomy of Abdomen' module. The batch was divided into 6 groups, each with 29-30 students. Two examiners conducted the viva for each group, 5 minutes per student in the presence of the whole group of students. Students were assessed using a structured set of questions with a marking scheme. Feedback was obtained in-writing and through an online questionnaire. Of the total 177 students who participated in the viva, $134(75.7 \%)$ students responded to the questionnaire. Majority of students were contented with the organization of viva (98.5\%), examiners' support (98.5\%), clarity (89.5\%) and relevance of questions (96.3\%), time allocated $(92.5 \%)$ and use of relevant material $(81.3 \%)$. Majority $(81.3 \%)$ was satisfied with the viva and $93.3 \%$ were motivated to study further. Thematic analysis of written feedback from all (177) the students revealed the use of viva to stimulate further learning (6.8\%), to improve knowledge (21.4\%) and communication skills (11.3\%), and for self-evaluation (9.6\%). Some students expressed its usefulness in future assessments $(15.3 \%)$, and to reduce nervousness (5.1\%). Viva was reported as stressful (14.7\%) and few disapproved its use for summative exams $(2.9 \%)$. The study demonstrated that, despite viva being a traditional assessment tool, when appropriately used it can be used to enhance learning by promoting internal motivation and providing opportunities for selfevaluation, knowledge and skills enhancement. 